\title{
Function Block Applications For Process Control System Based On Fieldbus Technology
}

\author{
Xia Zhuang \\ Civil Aviation Flight University of China, Sichuan, 618307 \\ 16405540@qq.com
}

Keywords: Process control system; Function block; Device; Standard.

\begin{abstract}
Present and future digital process control systems need to many requirements,for example,increase security and safety,reduce time to market, minimize training costs,support integrated methodology for implementation, and so on. Process control systems are required to fulfil these requirements in terms of their architecture and their operation during all the phases of the life cycle. The accepted basic concept for the design process control system is to describe all necessary implementation-specific functions with function block.IEC 61804 defines the function block for process control.In this paper, we discuss how a consistent function block capability may be provided for all fieldbus technology utilized in a control system. Examples will be given of how this standard has been applied in modern control systems to give a consistent interface to Foundation Fieldbus and PROFIBUS. Some detail will be presented on the standard means that is defined for manufacturers to describe function block capability of a field device.
\end{abstract}

\section{Introduction}

A variety of digital fieldbus devices and fieldbus technologies have been introduced within the process industry. There has been a gradually acceptance of the fact that a variety of communication technologies are needed to fully address the application requirements of a manufacturing facility. However, engineers responsible for the specification, engineer, and implement of control system require that a common interface and functionality be proved in the control system. This capability should be independent of the underlying fieldbus technology or manufacturer of the fieldbus device. The draft IEC 61804 standards defines how a control system can be structured to provide this flexibility in the utilization of fieldbus technology [1].

The draft IEC 61804 standards is an end user driven specification of the requirements of distributed process control systems based on Function Blocks. This requirement specification (which is defined as part 1) and its associated function block standard (part 2) originate from the power plant industrial sector. It is validated by applications in oil and gas, petrochemicals, pharma ceuticals and fine chemicals, pulp and paper, food and beverage, waste water treatment plants, steel milling and others. The specification defines the requirements for function blocks to provide control, and to facilitate the maintenance and the technical management as applications, which interact with actuators and measurement devices [2]. These parts were prepared by IEC SC65C WG7 and are available as draft IEC 61804.

\section{Fonction Block}

IEC 61804 defines a function block model which graphical representation is shown in Fig.1. It consists of the components FB Head and FB Body. The body carries the data flow (Data inputs and data outputs, algorithms, internal data and contained data, which are not involve in the data flow, but adjust the algorithms) and the head of the event of flow (event inputs, event outputs and execution control). 


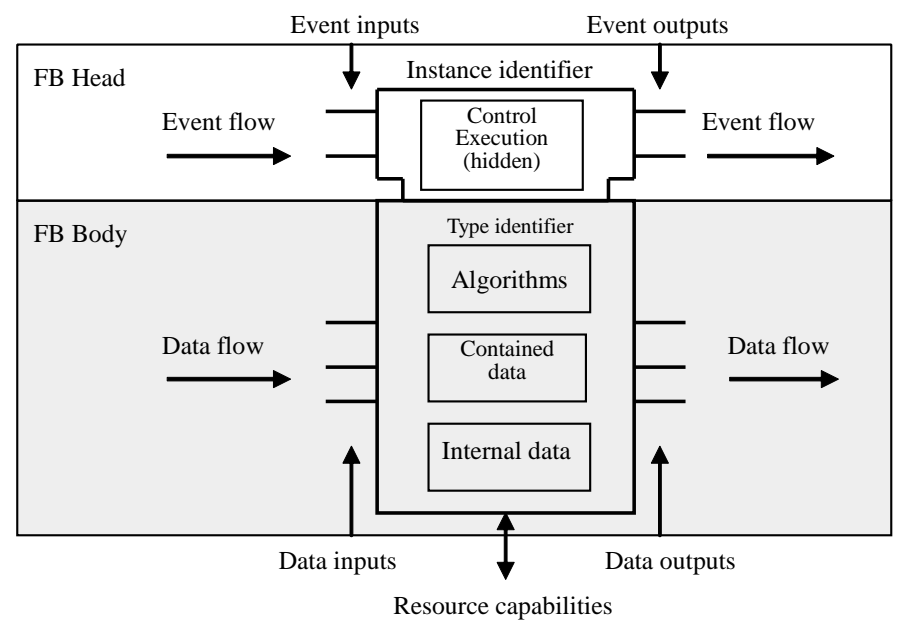

Figure 1. Structure of Function Block

The execution order of function blocks in a centralized program is determined by the order of the function block call in the program, or by the scheduling of the task system of one resource[3]. In the distributed environment in terms of IEC 61804 the execution order of function blocks is determined by the event flow of the FB heads. Because of the event connection between the execution control head of a function block and the local operating system which is seen as scheduling function. The head of the function block is the configurable part of the distributed operation system. In other words, the distributed operation system is build by the local operating system of each resource and the connections between function block execution control heads.

\section{Function Block Application Components}

From Control System to Function Block. Function Blocks are encapsulations of variables, parameters and their processing algorithms. The variables, parameters and algorithms are those required by the design of the process and its control system. They can be derived from the Pipe and Instrumentation Diagram (P\&ID)[4]. These are presented in 2 different clauses. One deals with "rich" FBs covering complex but common functions suchas control loop (for example proportional, integral, differential PID) required by the majority of the process industries. Another covers a set of elementary FBs (EFB) such as Boolean functions required to compose very specific and unique functionality.

The application can be distributed among several devices. The devices are connected via a communication network or a hierarchy of communication networks. There are different function block types, which encapsulate specific functionality of devices performing an automation application. The Technology Block represents the process attachment of a device. It contains the measurement or actuation principles of a device. The Application Function Block contains application related signal processing, such as scaling, alarm detection or control and calculation. Elementary functions and elementary function blocks contains mathematical and logical functions with specific additional exception handling procedures. The Device Block represents the resource of the device, which contains information and function about the device itself, the operation system of the device and the device hardware. 


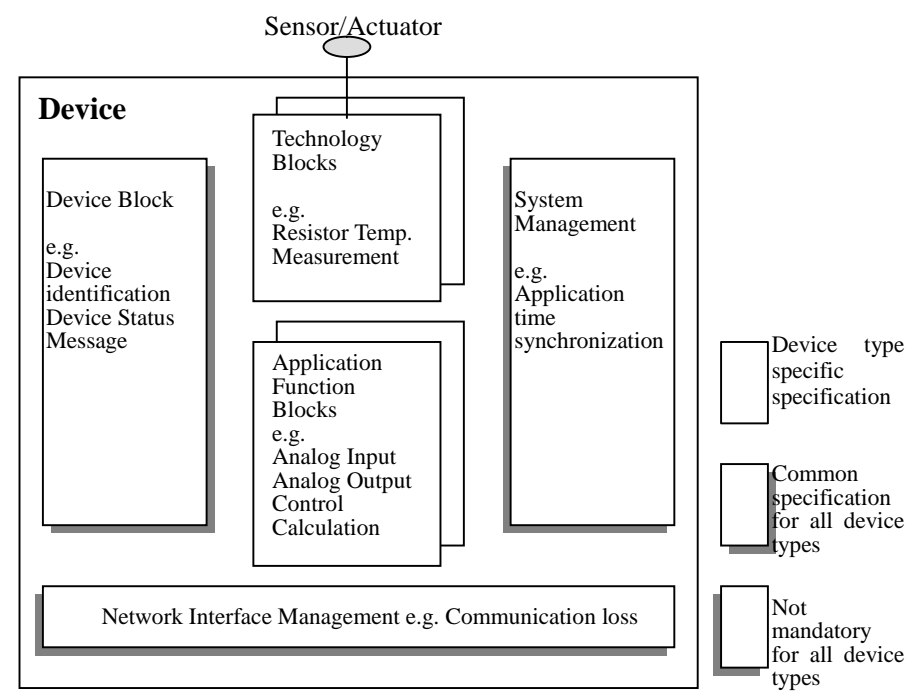

Figure 2. Device Structure

All devices in the scope of IEC 61804 are expected to have the same logical device structure. The number and types of blocks, which are instantiated in a device, are device and manufacturer specific. There is a data flow chain from signal detection through the Technology Block and Function Blocks and vice versa. The signals between the parts of the chain can be internal within the blocks or visible as linkages between blocks. The logical chain of technology and function block is called a channel.

IEC 61804 Function Block Overview. The description of an IEC 61804 function block is a list of algorithms that are encapsulated in the block together with the related input and output variables and parameters. There are algorithms that are related to the process signal flow and algorithms that are related to other block specific functions. The parameter table shows all the needed accessible parameters of the block.

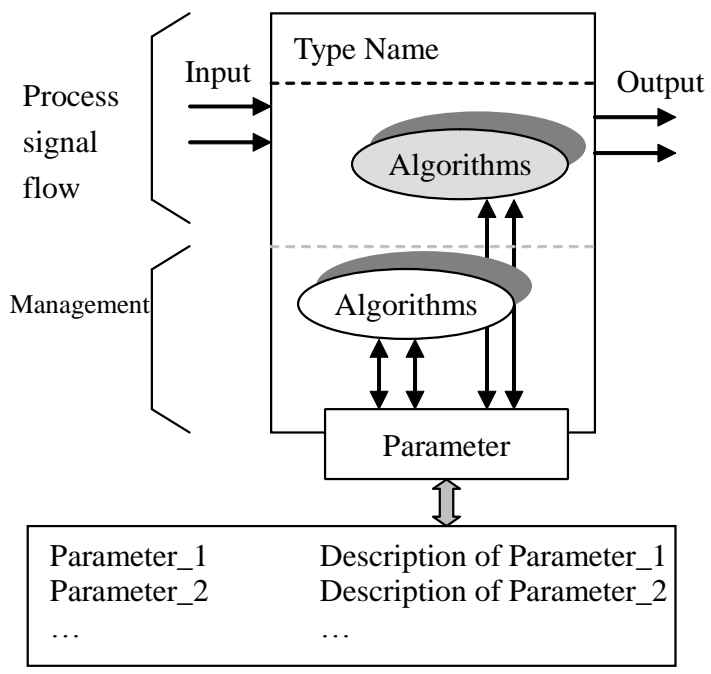

Figure 3. IEC 61804 function block overview

The algorithm description is done individual for each algorithm by a device designer/manufacturer in the appropriate language, e.g. plain English, Harel State Diagram, IEC 61131 FBD (function block diagram) or IEC 61131 ST (structured text). The block variables, parameters and algorithms included in a block will be those that are significant for the algorithm and device. As a minimum, function blocks will include the variables and parameters defined in the P\&ID. 


\section{Function Block Application}

The technology and application blocks built a function chain along with the process signal flow. Together they built a measurement or actuation channel. Measurement and actuation channels perform together with control and calculation function blocks in the application (Fig.4). The technology blocks are technology dependent and the function blocks are technology independent. There are different implementations of an application, depending from the used technology of the devices. The application may be performed by implementing the application in measurement and actuation devices only (i.e. a complex device may perform measurement, control and actuation.) or in measurement and actuation devices together with controllers and other components of the system. A controller may be for instance integrated in the application as one calculation function block or an actuation device may take parts of programmable functions from controller devices in terms of calculation function blocks.

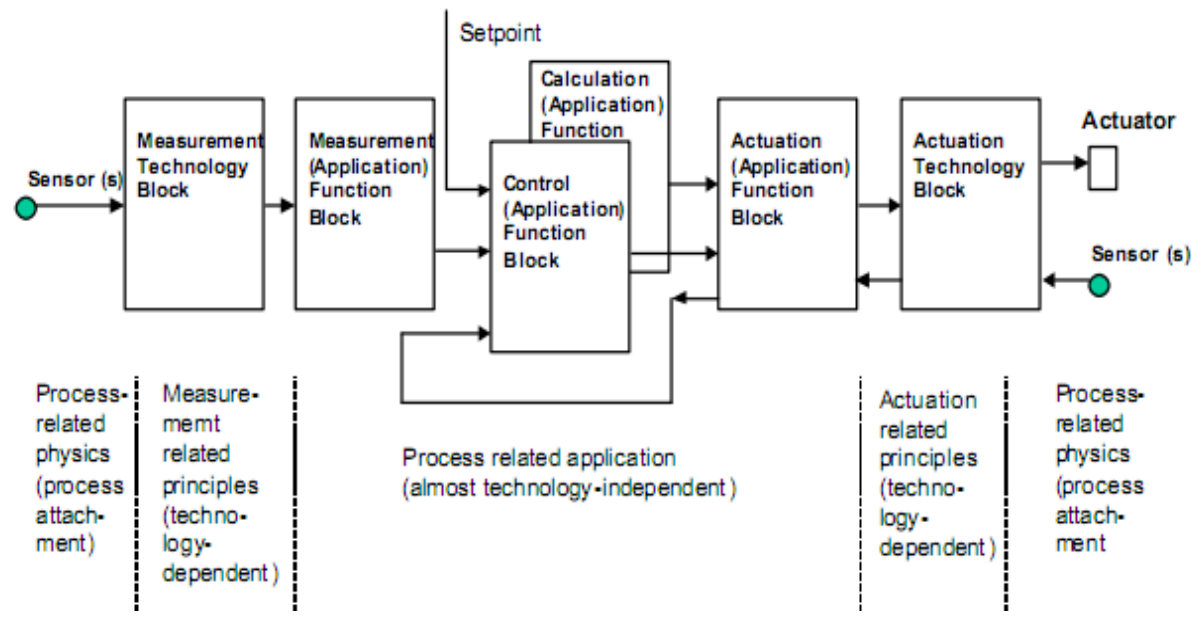

Figure 4. Application process signal flow

There are different device characteristics, called device types. The main characteristic aspect is the execution control methods. Execution control of function block algorithms is a feature of each device. There are many possible execution policies within devices and in a distributed system:

1. Free running

2. Device internal time schedule (time synchronization)

3. System wide time synchronization

4. Communication service triggered

5. Device internal event triggered

6. Distributed execution control

Which execution control is used depends on the capability of the devices. Therefore the execution control is defined by concrete fieldbus systems ${ }^{[5]}$.

\section{Example Application}

The function blocks resulting from the design of a process control system are abstract representations and may be implemented in different ways in different device types (Fig.5). Function blocks can be implemented e.g. in field devices, PLC, visualization stations and device descriptions. Additionally other applications such as system engineering and the supervisory system have to handle or interact with the function blocks. Functions defined for a function block in the conceptual model are not necessarily mapped one-to-one to the device; they can be mapped to the device and a proxy if the current technology doesn't solve it in the device. 


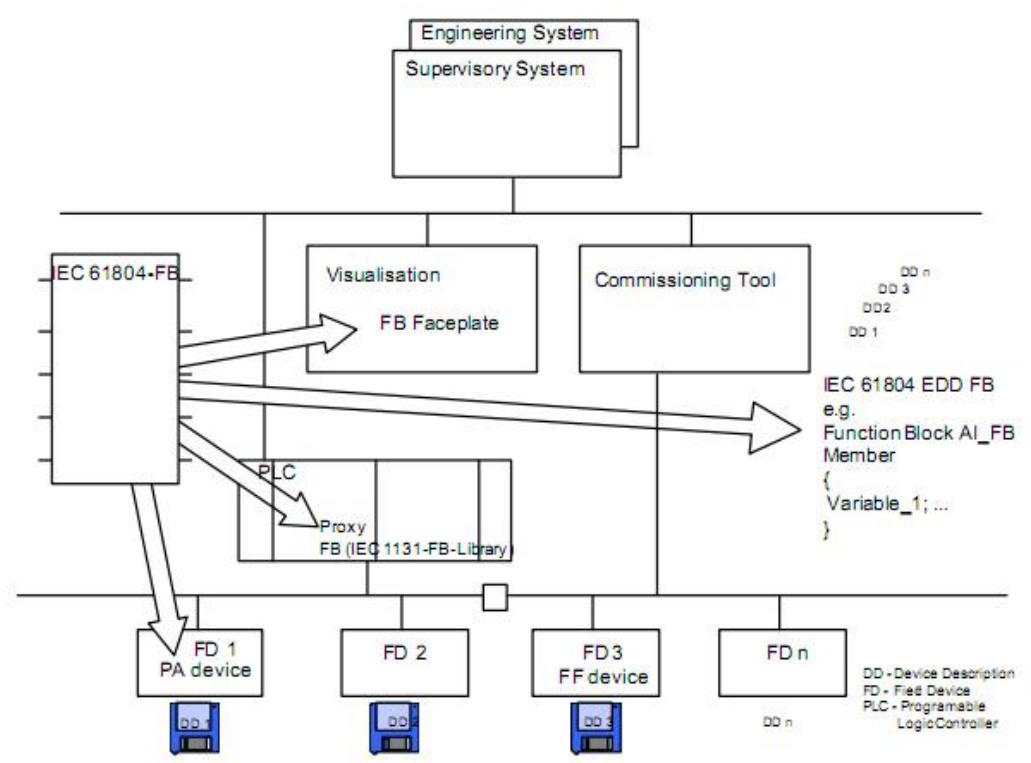

Figure 5. IEC 61804 function blocks can be implemented in different devices

One example of how the IEC 61804 specification may be applied to give a consistent function block interface to different technologies is the DeltaV Control system from Fisher-Rosemount Systems, Inc. The system architecture is based on Foundation Fieldbus function block specification and thus allows control strategies to be defined independent of whether the function block executes in the fieldbus device or in the DeltaV controller. Fieldbus devices that do not support function block such as those based on AS-Interface, Profibus DP, and DeviceNet are brought into the system using the concept of a proxy in the controller to add function block capability to the measurement or actuator value provided by the device.

To the end user, these proxy function blocks may be configured for monitoring and calculation application in the same manner as Foundation Fieldbus function blocks. The advanced signal processing and alarming that would normally be done by function blocks in the fieldbus device are done instead in the controller. Though the same functionality is provided through the proxy, there are differences in the dynamic associated with the signal processing. In many cases, these timing differences will have not impact on the application. An example configuration application that utilizes inputs and output from Foundation Fieldbus, AS-Interface, and Profibus DP is shown in Fig.6.

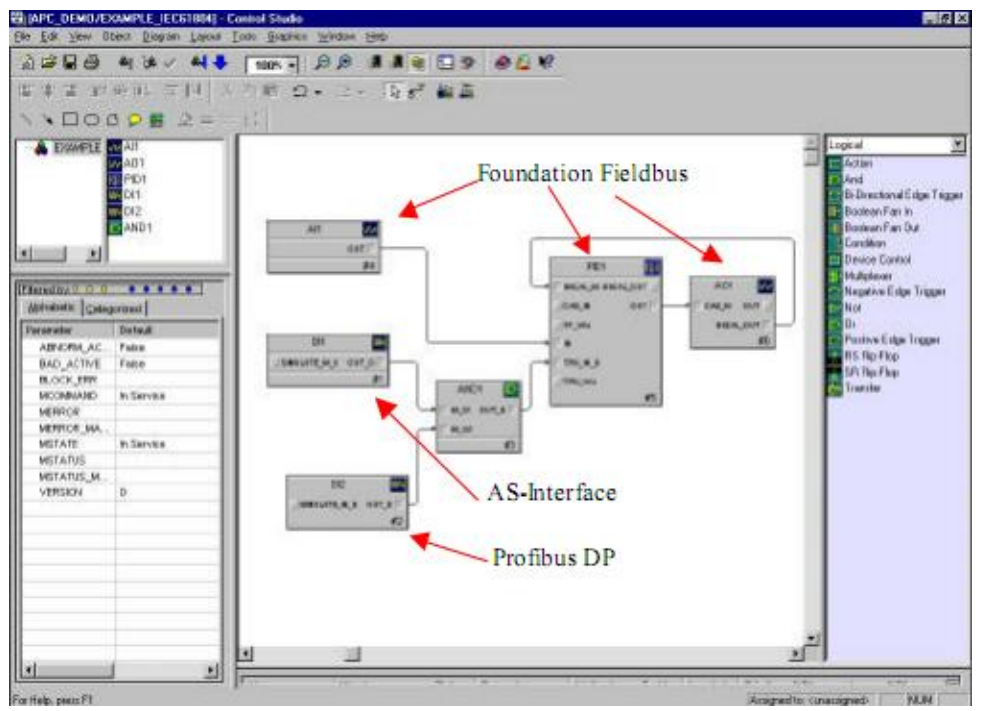

Figure 6. Function block example for Foundation Fieldbus, AS-Interface, and Profibus DP 


\section{Summary}

Process control systems that use fieldbus, function blocks and device descriptive languages already exist in the market. It is clear that the majority of these systems will continue to use function blocks into the foreseeable future, as they are modular and re-usable. However control system manufacturers, implementers and users are faced with:

- Multiple technology platforms that are changing rapidly.

- Multiple communication standards.

The approaches are similar, but the technologies are different.

As for the mapping on the several technologies, an important role is played by the proxy approach. It is expected that process plants users use the standard as a point of reference in specifying their control system requirements and in evaluating technology to meet these requirements in formal manner. Provided that some function blocks are not completely solved in the existing devices, the proxy approach allows the system integrator to complement these devices by solving the missing functionalities with some additional software installed in the controller. System integrators are able to define the proxies needed for the different applications and for the different industrial sectors. Thus IEC 61804 allows the users to carry a top-level view of their control systems forward into the future and the manufacturers to answer with evolving solutions.

\section{References}

[1] IEC 61804-Function blocks (FB) for process control Part 1: General Requirements Committee Draft, International Electric Commission, Draft, Tech. Comm. 65, working group 6, Geneva

[2] IEC 61804-Function blocks (FB) for process control Part 2: Specification Committee Draft, International Electric Commission, Draft, Tech. Comm. 65, working group 6, Geneva

[3] Brennan,R.Zhang,X.and Norrie,H. A Reconfigurable Concurrent Function Block Model and its Implementation in Real-Time Java. Journal of Integrated Computer-Aided Engineering [J], 2011, PP263-279.

[4] Chen,Hsin-Chieh. Chang, Jen-Fuh.EP-based PID control design for chaotic synchronization with application in secure communication. Expert Systems with Applications[J] ,2013.2,PP1169-1177.

[5] Mahalik, N.P.Yen,Matthew.Extending fieldbus standards to food processing and packaging industry. Computer Standards \& Interfaces[J]. 2012.3, PP586-598.

[6] D.Chivilikhin, A.Shalyto.Inferring Automata Logic from Manual Control Scenarios: Implementation in Function Blocks [J]. 2015.3, PP211-218.

[7] J.Lahtinen. Automatic Test Set Generation for Function Block Based Systems Using Model Checking. 2014.8, PP117-124.

[8] D.Kleyko, E.Osipov,Matthew.On methodology of implementing distributed function block applications using TinyOS WSN nodes [J]. 2014.5, PP326-331.

[9] P.Mani, M.Prasanna.Automatic test case generation for programmable logic controller using function block diagram [J]. 2016.3, PP401-408. 